\title{
Changes in functional brain networks and neurocognitive function in Chinese gynecological cancer patients after chemotherapy: a prospective longitudinal study
}

Yingchun Zeng ${ }^{1,2}$, Andy S. K. Cheng ${ }^{2^{*}}$, Ting Song ${ }^{3}$, Xiujie Sheng ${ }^{1}$, Huaidong Cheng ${ }^{4^{*}}$, Yingwei Qiu ${ }^{3}$, Jianfei $\mathrm{Xie}^{5}$ and Chetwyn C. H. Chan ${ }^{2}$

\begin{abstract}
Background: Previous neurocognitive assessments in non-central nervous system cancers highlight the high incidence of neurocognitive dysfunction in this study population. However, there have been few studies exploring neurocognitive dysfunction induced by chemotherapy in gynecological cancer patients. This prospective longitudinal study was conducted to assess neurocognitive functioning and functional brain networks in Chinese gynecological cancer patients pre- and post-chemotherapy, while additionally including age-matched healthy subjects as the control group.

Methods: All research participants were evaluated using a resting-state functional magnetic resonance imaging and neurocognition assessment. Behavioral data were conducted using SPSS for descriptive statistics, correlation and comparison analyses. Preprocessing of MRI (Magnetic Resonance Imaging) data and network analyses were performed using GRETNA (Graph Theoretical Network Analysis).

Results: A total of 40 subjects joined this study, with 20 subjects in each group. With the exception of the mean of psychomotor speed, there was no significant difference pre-chemotherapy between patients and healthy controls in neurocognitive test mean scores (Ps > 0.05). During the post-chemotherapy assessment, there were significant differences in the mean scores of neurocognitive tests (including Digit Span tests, verbal memory, immediate recall, delayed recall, and information processing speed tests) (all Ps $<0$.05). Longitudinal graph analysis revealed statistically significant differences in the patient group, with significant decreases in both local efficiency $(P<0.01)$ and global efficiency $(P=0.04)$. Lower raw TMT-A scores were significantly associated with lower local efficiency $(r=0.37, P=0.03)$. Lower verbal memory scores were statistically significant and associated with lower global efficiency $(r=0.54, P=0.02)$ in the patient group, but not in the healthy control group.

Conclusions: This study found that the risk of brain function and neurocognitive changes following chemotherapy could potentially guide patients in making appropriate treatment decisions, and this study may identify a cohort that could be suited for study of an intervention.
\end{abstract}

Keywords: Gynecological cancer, Chemotherapy, Neurocognitive function, Functional brain networks, Chinese patients

\footnotetext{
*Correspondence: andy.cheng@polyu.edu.hk; 596830447@qq.com

${ }^{2}$ Department of Rehabilitation Sciences, The Hong Kong Polytechnic

University, Hung Hom, Hong Kong, China

${ }^{4}$ Department of Oncology, The Second Affiliated Hospital of Anhui Medical

University, Hefel, China

Full list of author information is available at the end of the article
}

(c) The Author(s). 2019 Open Access This article is distributed under the terms of the Creative Commons Attribution 4.0 International License (http://creativecommons.org/licenses/by/4.0/), which permits unrestricted use, distribution, and

reproduction in any medium, provided you give appropriate credit to the original author(s) and the source, provide a link to the Creative Commons license, and indicate if changes were made. The Creative Commons Public Domain Dedication waiver (http://creativecommons.org/publicdomain/zero/1.0/) applies to the data made available in this article, unless otherwise stated. 


\section{Background}

Gynecological cancer is the third most common malignancy among women in China [1]. While improving early cancer diagnosis and accessing effective cancer treatment increase cancer patients' five-year relative survival rate, neurocognitive dysfunctions are significant sequelae of cancer [2]. Neurocognitive dysfunctions may affect executive function, psychomotor speed, attention and memory [3]. This can interfere with gynecological cancer patients' capacity to accomplish activities of daily living, as well as with social and occupational functioning, leading to lower quality of life $[2,4,5]$.

Advanced neuroimaging studies in cancer patients provide a better understanding of neurocognitive dysfunction after cancer treatment [6]. Previous neuroimaging studies have indicated changes in brain structure and function that correlate with neurocognitive function in gynecological cancer patients [7, 8]. While multiple neuroimaging studies have demonstrated structural and functional brain differences between cancer patients and healthy controls [9], structural changes in the brain cannot serve as a prompt or reliable biomarker for early diagnosis of treatment-induced neurocognitive disorders [10], as abnormalities in brain function usually appear before alterations in brain structure and clinical performance [11]. Certainly, some studies have demonstrated that structural changes co-occur with functional network differences [12], and the structure-function relationship is modality dependent [13]. Other research conducted on cancer patients also found that disruptions in brain structure and/or function may parallel [14]. Hence, detecting alterations in structural or functional brain networks might provide an earlier biomarker for neurocognitive dysfunction diagnosis [10].

Functional magnetic resonance imaging (fMRI) studies have shown that quantitative neuroimaging techniques, in combination with neurocognitive assessment, can be useful in advancing our understanding of treatment-induced neurocognitive dysfunction in cancer patients [7, 14, 15]. Different from task-dependent fMRI studies, resting state fMRI (Rs-fMRI) is task-independent and thus less vulnerable to confounds due to performance variance [16]. Rs-fMRI is a noninvasive neuroimaging technique that measures spontaneous brain activity [17]. Rs-fMRI does not require participants to engage in any cognitive activity, therefore providing unique advantages for clinical research studies $[18,19]$. In addition, rs-fMRI is sensitive enough to measure intrinsic functional networks, which reflect various cognitive states, representing the majority of energy usage in the brain [20]. Thus, using rs-fMRI to detect brain changes pre- and postchemotherapy is likely associated with aspects of disease and treatment pathology for cognitive dysfunction [14]. And utilizing a network analysis of rs-fMRI data, and linked neurocognitive changes with functional brain networks in cancer patients, would be promising to address the issue of interest in the present study.

The majority of neuroimaging studies on the neurocognitive functioning of patients treated with chemotherapy for non-central nervous system cancers have been conducted on breast cancer patients [10, 15]. Limited neuroimaging studies have been conducted on patients with gynecological cancer [7, 8, 21-23]. Given the poor understanding of the impacts of cancer and its treatment on neurocognitive function and functional brain networks in gynecological cancer patients, particularly Chinese cancer patients, it is important to explore any neurocognitive changes or functional brain network alterations in this population. Therefore, this prospective longitudinal study was conducted to assess the neurocognitive function, and functional brain networks, of Chinese gynecological cancer patients pre- and postchemotherapy. The findings could add to the literature in meaningful ways by studying a cancer type that has received limited attention in terms of the cognitive and neuroimaging effects of treatment, while adding to the small body of literature that has examined these issues in non-Caucasian patient groups.

\section{Methods \\ Subjects}

The details of the study subjects have been previously described [24] but briefly described as Chinese adult women with a primary diagnosis of cervical, ovarian, or uterine cancer were ready for chemotherapy treatment. Age-matched women without cancer history were recruited as healthy controls. All age-matched healthy controls were recruited from among staff members at this hospital. This study obtained ethical approval from the ethics committees at both The Hong Kong Polytechnic University and The Third Affiliated Hospital of Guangzhou Medical University. All research participants joined this study voluntarily and provided written informed consent.

\section{Neurocognitive function assessment}

The details of neurocognitive function assessment have been previously described [24] but briefly summarized as follow: this study took the recommendation of the International Cognition and Cancer Task Force (ICCTF): three core neurocognitive tests of the Hopkins Verbal Learning Test - revised (HVLT-R), the Trail Making Test (TMT), and the Controlled Oral Word Association Test (COWA) were used [25]. This study used the Chinese version of the Auditory Verbal Learning Test - revised version (AVLT-R) [26]; the Chinese version of TMT and the Chinese version of the COWA [27], respectively. As attention and working memory were the most common 
neurocognitive dysfunctions in Chinese gynecological cancer patients [28], this study also included the WAIS-III Digit Span test for measuring attention and working memory [29]. Cognitive performance testing in the patient group was conducted at post-surgery and post-chemotherapy, respectively. The duration of cognitive performance tests for patients were four months. As recruiting healthy controls needs to be age-matched with patients, it took two months to recruit eligible healthy controls, the duration of cognitive performance tests for healthy controls was two months. AVLT tests consisted of three successive learning trials and other procedures of cognitive tests were conducted consistently in both groups.

\section{MRI data acquisition}

According to the ICCTF recommendations for neuroimaging studies in cancer and cognition, a minimal set of MRI sequences should include an rs-fMRI and a high-resolution T1-weighted anatomical MRI scan to assess functional brain networks [6]. Whole brain rs-fMRI data were collected on a Philips 3.0 T scanner (Achieva; Philips, Best, The Netherlands), using an 8-channel SENSE head coil at The Third Affiliated Hospital of Guangzhou Medical University, China. Throughout the rs-fMRI data acquisition, patients were instructed to close their eyes and relax, but to remain in a maximally alert state. A T2-weighted gradient-echo EPI sequence was used to obtain the rs-fMRI scan. A total of 240 whole brain EPI volumes were acquired using the following parameters: $\mathrm{TR}=2000 \mathrm{~ms}, \mathrm{TE}=30 \mathrm{~ms}$, flip angle $=90^{\circ}$, in-plane imaging resolution $=3 \times 3 \times 3 \mathrm{~mm}$, in-plane field of view $(\mathrm{FOV})=256 \times 256 \mathrm{~mm}$, slice thickness $=4 \mathrm{~mm}$, axial slices $=33$. The rs-fMRI scan time was $8 \min 6 \mathrm{~s}$. T1-weighted imaging was achieved for morphometric (GM volume, cortical thickness and surface area) analysis using three-dimensional fast spoiled-gradient recalled acquisition in steady state (3D-FSPGR) in 164 coronal slices with the following parameters: acquisition matrix $=256 \times$ 256; $\mathrm{TE}=3.8 \mathrm{~ms} ; \mathrm{TR}=8.2 \mathrm{~ms}$; flip angle $=7^{\circ} ; \mathrm{FOV}=256$ $\mathrm{mm} \times 256 \mathrm{~mm}$; slice thickness $=1 \mathrm{~mm}$; voxel resolution $=1 \times 1 \times 1 \mathrm{~mm}$. The 3D-T1 scanning time was $5 \mathrm{~min} 58 \mathrm{~s}$.

\section{MRI data preprocessing and network analyses}

The rs-fMRI images were preprocessed using GRETNA: a graph theoretical network analysis toolbox for imaging connectomes [30]. During the preprocessing process, the first 10 volumes for signal were removed to reach a steady state, leaving 230 functional volumes for each subject. The remaining functional volumes were corrected for acquisition time delay between slices (slice timing) and head motion between volumes (realignment). Other steps in preprocessing these functional data consisted of spatial normalizing by DARTEL (warping individual functional images to the standard MNI space by applying the transformation matrix that can be derived from registering the final template file), spatially smoothing with a Gaussian kernel (full width at half-maximum of $4 \mathrm{~mm}$ ), regressing out covariates (white matter, cerebral spinal fluid, global signals, and head-motion profiles are removed to avoid noise signals by multiple regression analysis), temporally linear detrending, temporal band-pass filtering $(0.01-0.1 \mathrm{~Hz})$, and scrubbing to reduce the effects of head motion on rs-fMRI data. The networks are constructed based on a voxel or region of interest approach. The Automated Anatomical Labeling (AAL) atlas was used to parcellate the brain into 90 regions (cerebellum excluded). Functional brain networks were constructed by thresholding the correlation matrices with a density of $5 \%$ [31]. All network analyses were performed using GRETNA [30]. The values were mapped onto the cortical surface using BrainNet Viewer [32]. Data preprocessing and network analyses are shown in Fig. 1.

\section{Hub identification}

There are various methods to identify functional hubs. Some research suggests that hub regions can be defined as degree, betweenness centrality, and/or clustering coefficient values exceeding $1 \mathrm{SD}$ (Standard Deviation) above the mean network, thus indicating hub status [33]. Other research indicates that nodes with a high degree, exceeding 1.5 SD above the mean network, can be identified as functional hubs, meaning that they exhibit high connectivity to the rest of the brain [31]. This study defined functional hubs of research participants with node degree values exceeding $1.5 \mathrm{SD}$ above the mean network.

\section{Statistical analysis}

Descriptive statistics, correlation and comparison analyses were conducted using SPSS for Windows (version 21; IBM SPSS Statistics, Armonk, NY, U.S.). Descriptive statistics are presented as mean, standard deviation (SD), and range. According to ICCTF recommendations, cancer patients were rated as experiencing cognitive dysfunction "if two or more neurocognitive tests (AVLT, TMT, COWA and Digit Span test) had a Z-score at or below -1.5 , and/or one test had a Z-score at or below -2.0 of the healthy control group" [25]. Transformation of Z-scores was computed as subjects' raw score minus the mean group score and divided by the standard deviation. Correlations of neurocognitive function with brain functional connectivity were made using Pearson correlation coefficients. Group differences were tested with independent or paired t-tests. All statistical tests performed were two-sided, and a $P$ value of less than 0.05 was considered statistically significant. 


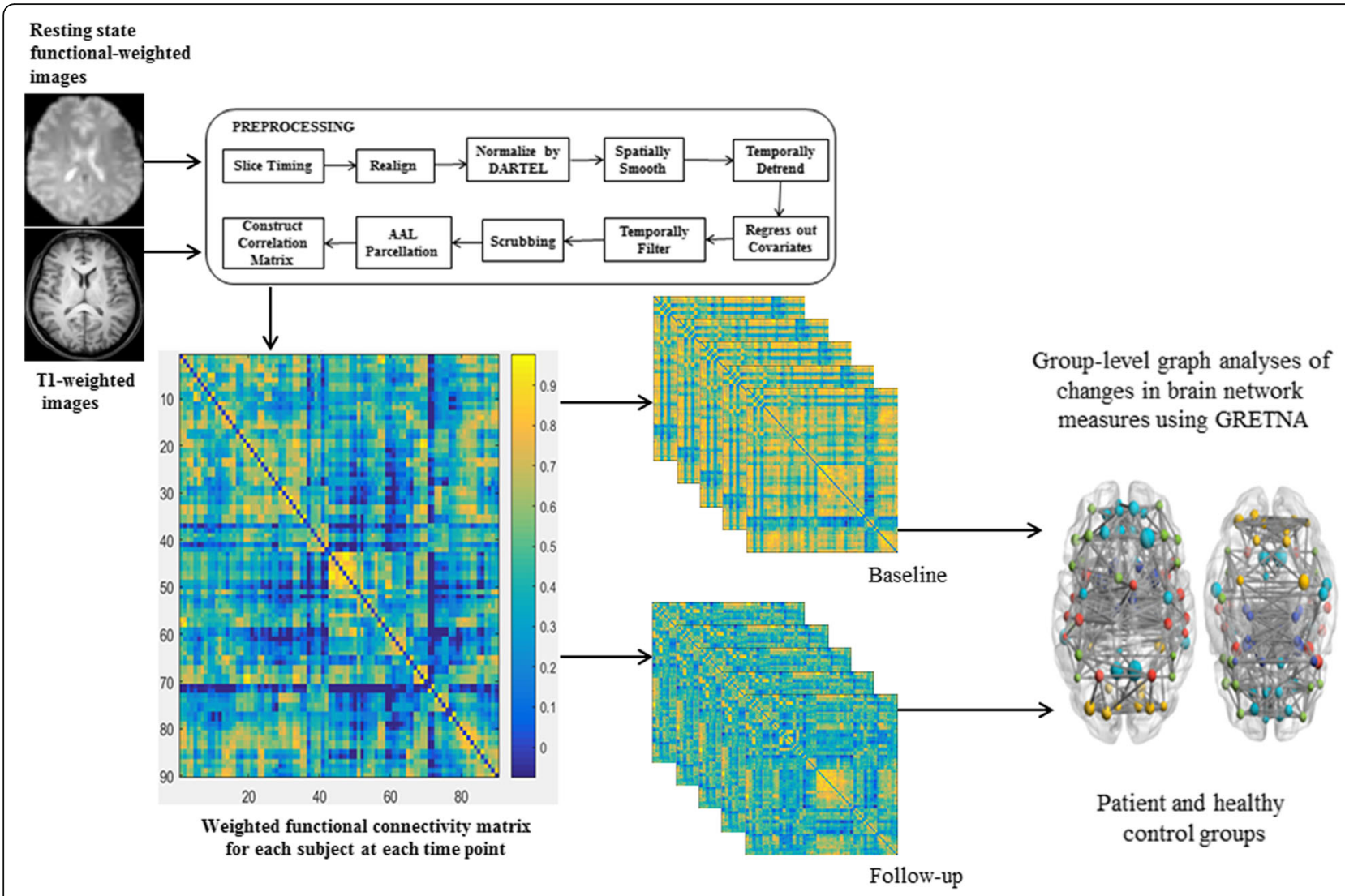

Fig. 1 Illustration of brain functional network construction for longitudinal graph analysis

\section{Validation analysis}

To evaluate the robustness or reproducibility of the result, this study modified two key parameters of global signal regression and different connectivity density thresholds. For the validation of correlation between functional global metrics and associations with neurocognitive outcomes, this study used partial correlation analysis to adjust global signal as the covariate, as global signal of the whole brain is an important confounding factor for brain network analyses based on rs-fMRI [34]. Taken consideration of the connectivity density as an important parameter of the network topological structure, this study also validated study findings with network densities of 3 and $7 \%$ suggested by previous research [31]. A network null model for each group was designed with a density of $5 \%$ for comparison, as the weights of connections survived after thresholding with a density of $5 \%$ were applied.

\section{Results}

\section{Research participant characteristics}

Of 37 eligible patients, a total of 20 patients agreed to join this study and completed the baseline rs-fMRI and neurocognitive assessment. Four patients refused to attend the MRI scans and neurocognitive assessment post-chemotherapy. There were 20 healthy control subjects who were matched in terms of age, marital and menopausal status. The demographic and clinical characteristics of the research participants are summarized in Table 1.

\section{Neurocognitive function of cancer patients compared to healthy controls}

As illustrated in Table 2, with the exception of information processing speed, there was no significant difference at T1 (pre-chemotherapy) in the neurocognitive test mean scores between patients and healthy controls (Ps $>0.05$ ). There was a significant difference in neurocognitive test scores, (including Digit Span tests, AVLT immediate recall and delayed recall, and TMT-A) (all Ps $<0.05$ ) at T2 (post-chemotherapy). Transformation of patient Z-scores was computed as patients' raw score minus the mean of the control group score at $\mathrm{T} 1$ and divided by $\mathrm{SD}$. Z-scores of cognitive tests at $\mathrm{T} 1$ and $\mathrm{T} 2$ adjusted for education and employment status. From Table 3, there was a significant difference in $\mathrm{Z}$ scores of neurocognitive tests (including Digit Span tests, AVLT and TMT-A) (all Ps $<0.05$ ) between patients and healthy controls at $\mathrm{T} 1$ and $\mathrm{T} 2$. There were seven patients at $\mathrm{T} 1$ and nine patients at $\mathrm{T} 2$ who reported cognitive dysfunction, respectively (Table 4). 
Table 1 Demographic and clinical characteristics $(N=40)$

\begin{tabular}{|c|c|c|}
\hline \multirow[t]{2}{*}{ Variables } & \multicolumn{2}{|l|}{$\mathrm{n}(\%)$} \\
\hline & $\begin{array}{l}\text { Cancer patients } \\
(n=20)\end{array}$ & $\begin{array}{l}\text { Healthy controls } \\
(n=20)\end{array}$ \\
\hline Age (years) Mean (SD) & 47.15 (9.80) (28-60) & $48.60(6.80)(29-59)$ \\
\hline \multicolumn{3}{|l|}{ Highest education } \\
\hline Primary school or below & $14(70.0)$ & $19(95.0)$ \\
\hline High school & $4(20.0)$ & $1(5.0)$ \\
\hline University and above & $2(10.0)$ & \\
\hline \multicolumn{3}{|l|}{ Employment status } \\
\hline Employed & $1(5.0)$ & $20(100)$ \\
\hline Unemployed & $19(95.0)$ & \\
\hline \multicolumn{3}{|l|}{ Marital status } \\
\hline Never married & $2(10.0)$ & $1(5.0)$ \\
\hline Married & $18(90.0)$ & $18(90.0)$ \\
\hline Divorced & $0(0.0)$ & $1(5.0)$ \\
\hline \multicolumn{3}{|l|}{ Menopausal status } \\
\hline Pre-menopausal & $11(55.0)$ & $12(60.0)$ \\
\hline Peri-menopausal & $8(40.0)$ & $7(35.0)$ \\
\hline Post-menopausal & $1(5.0)$ & $1(5.0)$ \\
\hline \multicolumn{3}{|l|}{ Cancer type } \\
\hline Cervical cancer & $8(40.0)$ & \\
\hline Ovarian cancer & $5(25.0)$ & \\
\hline Uterine cancer & $7(35.0)$ & \\
\hline \multicolumn{3}{|l|}{ Disease stage } \\
\hline Stage I-Ila & $7(35.0)$ & \\
\hline Stage IIb-IIla & $8(40.0)$ & \\
\hline Stage IIIb & $5(25.0)$ & \\
\hline \multicolumn{3}{|l|}{ Treatment type } \\
\hline Surgery + Chemotherapy ${ }^{a}$ & $14(70.0)$ & \\
\hline $\begin{array}{l}\text { Surgery }+ \text { Chemotherapy }^{\mathrm{a}}+ \\
\text { Radiation }\end{array}$ & $6(30.0)$ & \\
\hline
\end{tabular}

${ }^{a}$ Chemotherapy regimens including Paclitaxel (TAXOL) with Carboplatin (CBP) or Cisplatin (DDP) or with both; CBP with Doxorubicin (ADM) or TAXOL with ADM; Bleomycin with Methotrexate (MTX) or MTX with DDP

\section{Brain functional global metrics and associations with neurocognitive outcomes}

All participants in the patient and healthy control groups demonstrated a small-world organization as indicated by small-worldness greater than 1 . There were significant differences in small-worldness at T1 and T2 between patients and healthy controls $(P=0.04$, and $P=$ 0.02 , respectively) (Table 5 ). There was a significant increase in characteristic path length at T2 between patients and healthy controls $(P=0.01)$. Results from the longitudinal graph analysis revealed a reducing trend of local and global efficiency in the patient group (Table 5). Lower raw TMT-A scores were significantly associated with lower local efficiency $(r=0.28, P=0.04)$, and lower verbal memory scores were statistically significant and associated with lower global efficiency $(r=0.41, P=$ 0.03) in the patient group, but not in the healthy control group.

\section{Characteristics of hub brain regions related to neurocognitive dysfunction}

Brain regions of research participants were evaluated for network hub status based on nodal degree values exceeding 1.5 SD above the mean network [31]. Hub characteristics of brain regions are shown in Figs. 2 and 3. As seen in Fig. 2, functional hub brain regions for cancer patients are mainly located in temporal regions, while parietal regions are the functional hubs in healthy controls. Within the patient group, left hippocampus, left parahippocampal gyrus, left and right insula; middle temporal gyrus, and superior temporal gyrus are functional hubs for patients with neurocognitive dysfunction (Fig. 3).

\section{Discussion}

This is the first study to include a healthy control group with similar demographic characteristics, and a longitudinal design with repeated rs-fMRI assessment with the application of a longitudinal graph theoretical approach, to analyze brain functional networks in Chinese gynecological cancer patients. This study found that after chemotherapy treatment, gynecological cancer patients had lower neurocognitive test performance and changes in functional network measures, compared to age-matched healthy controls, which was in line with previous studies on cancer patients after chemotherapy $[10,14]$. But the mean score changes of cognitive tests in the patient group were small in this study. It may be possibly due to surgery-related cognitive impairment, as all patients at baseline received treatment of surgery. Other research suggests that cancer patients treated with local surgery yield larger cognitive impairment than patients' own baseline [35]. In specific, disrupted smallworld properties were found in gynecological cancer patients. Functional networks with prominent small-world properties ensure higher information-processing efficiency for both locally specialized and globally integrated processing [36]. Decreased small-worldness index among cancer patients may result in lower information processing speed, which was supported by the significant associations of lower local network efficiency with lower raw TMT-A scores.

While the findings of this study indicated that the functional brain networks of both cancer patients and healthy controls show common small-world properties (both groups' index values $>1$ ), the local efficiencies were significantly higher in cancer patients post-chemotherapy than in the healthy controls. As local efficiency is a measure 
Table 2 Comparison of cognitive testing mean scores between patients and healthy controls at T1 and T2

\begin{tabular}{|c|c|c|c|c|c|c|}
\hline \multirow[t]{2}{*}{ Variables } & \multicolumn{2}{|c|}{ T1 Mean (Standard Deviation-SD) } & \multirow[t]{2}{*}{$P$} & \multicolumn{2}{|l|}{ T2 Mean (SD) } & \multirow[t]{2}{*}{$P$} \\
\hline & Patients $(n=20)$ & Healthy Controls $(n=20)$ & & Patients $(n=16)$ & Healthy Controls $(n=16)$ & \\
\hline \multicolumn{7}{|c|}{ Attention and working memory } \\
\hline Digit span forward & $6.75(2.53)$ & $7.30(1.92)$ & 0.44 & $6.65(2.55)$ & $7.53(2.03)$ & 0.02 \\
\hline Digit span backward & $2.45(1.43)$ & $3.15(2.30)$ & 0.25 & $2.40(1.69)$ & $4.26(2.23)$ & $<0.01$ \\
\hline \multicolumn{7}{|l|}{ Verbal memory } \\
\hline AVLT immediate recall & $5.32(1.72)$ & $4.63(1.41)$ & 0.43 & $5.10(1.88)$ & $9.15(3.71)$ & $<0.01$ \\
\hline AVLT delayed recall & $4.95(2.58)$ & $4.45(2.32)$ & 0.52 & $5.01(2.92)$ & $7.35(2.34)$ & 0.01 \\
\hline AVLT recognition & $10.35(1.72)$ & $10.40(1.46)$ & 0.92 & $9.55(3.21)$ & $10.40(1.75)$ & 0.31 \\
\hline \multicolumn{7}{|c|}{ Information processing speed } \\
\hline TMT-A & $57.65(21.65)$ & $44.95(16.01)$ & 0.04 & $54.20(19.02)$ & $38.83(25.53)$ & 0.04 \\
\hline \multicolumn{7}{|l|}{ Executive function } \\
\hline TMT-B & $71.05(26.94)$ & $57.80(21.30)$ & 0.09 & $73.35(29.40)$ & $56.72(33.95)$ & 0.11 \\
\hline \multicolumn{7}{|l|}{ Language } \\
\hline COWA & 33.65 (8.89) & 31.55 (6.48) & 0.31 & 15.55 (5.96) & $25.65(22.18)$ & 0.36 \\
\hline
\end{tabular}

Abbreviation: AVLT Auditory Verbal Learning Test, COWA Controlled Oral Word Association Test, TMT Trail Making Test

of average local subgraphs in a network, increasing local efficiency in cancer patients may result in disrupted information processing among distant brain regions [37], and lower network attack tolerance was associated with greater neurocognitive dysfunction in cancer patients [14]. In addition, this study found significantly decreased global efficiency, and significantly positive correlations between decreased global efficiency and lower memory scores, in the patient group only. Study findings were consistent with previous research, which reported reduced functional brain network efficiency in response to a simulated neurodegeneration in breast cancer

Table 3 Cognitive testing Z-scores between T1 and T2 among cancer patients

\begin{tabular}{|c|c|c|c|}
\hline & $\begin{array}{l}\text { Patients at T1 } \\
(n=16)\end{array}$ & $\begin{array}{l}\text { Patients at T2 } \\
(n=16)\end{array}$ & $P$ \\
\hline \multicolumn{4}{|c|}{ Attention and working memory } \\
\hline Digit span forward & $-0.22(1.16)$ & $-0.32(0.45)$ & 0.04 \\
\hline Digit span backward & $-0.25(1.28)$ & $-0.08(1.39)$ & 0.02 \\
\hline \multicolumn{4}{|l|}{ Verbal memory } \\
\hline AVLT immediate recall & $0.81(1.05)$ & $-0.11(1.10)$ & $<0.01$ \\
\hline AVLT delayed recall & $0.72(0.86)$ & $-0.48(1.16)$ & $<0.01$ \\
\hline AVLT recognition & $0.42(0.93)$ & $-0.52(1.11)$ & $<0.01$ \\
\hline \multicolumn{4}{|c|}{ Information processing speed } \\
\hline TMT-A & $-0.14(1.07)$ & $-0.20(1.08)$ & 0.03 \\
\hline \multicolumn{4}{|l|}{ Executive function } \\
\hline TMT-B & $-0.19(1.08)$ & $-0.15(1.02)$ & 0.18 \\
\hline \multicolumn{4}{|l|}{ Language } \\
\hline COWA & $0.02(0.88)$ & $-0.04(0.99)$ & 0.13 \\
\hline
\end{tabular}

Abbreviation: AVLT Auditory Verbal Learning Test, COWA Controlled Oral Word Association Test, TMT Trail Making Test

Z-scores adjusted for education and employment status survivors receiving chemotherapy, compared with healthy controls [15].

This study found that functional hubs were mostly located in the temporal regions for patients, and in the frontal and parietal regions for healthy controls, reflecting the main functions associated with these brain regions [36]. These study findings discriminated between the functional hub networks of patients and those of healthy controls, and also identified functional hubs for patients with cognitive dysfunction as well as for patients without cognitive dysfunction. Functional hubs for patients with cognitive dysfunction included the left and right insula, middle temporal gyrus, superior temporal gyrus, left hippocampus and parahippocampal gyrus, which are essential for network resilience and regulation of information flow [38], as functional hubs play key roles in forming bridges between different networks [39]. Brain regions with a high node degree were identified as hubs, which would be the most vulnerable areas in local functional networks [15]. Taken together, these findings suggest that all of these hub brain regions are key regions implicated in the pathophysiology of

Table 4 Frequency of patients with decline on cognitive dysfunctions at $\mathrm{T} 1$ and $\mathrm{T} 2$

\begin{tabular}{lll}
\hline Cognitive domains & $\begin{array}{l}\text { Patients with cognitive dysfunctions } \\
\text { (No. \%) }\end{array}$ \\
\cline { 2 - 3 } & T1 (n=20,35.00\%) & T2 (n=16, 56.25\%) \\
\hline Attention and working memory & $2(10.00)$ & $3(18.75)$ \\
Verbal and learning memory & $2(10.00)$ & $3(18.75)$ \\
Information processing speed & $1(5.00)$ & $1(6.25)$ \\
Executive function & $1(5.00)$ & $1(6.25)$ \\
Verbal fluency & $1(5.00)$ & $1(6.25)$ \\
\hline
\end{tabular}


Table 5 Comparison of functional global metrics between patients and healthy controls at T1 and T2

\begin{tabular}{|c|c|c|c|c|c|c|}
\hline & $\mathrm{T} 1$ & & $P$ & $\mathrm{~T} 2$ & & $P$ \\
\hline & Patients $(n=20)$ & Healthy Controls $(n=20)$ & & Patients $(n=16)$ & Healthy Controls $(n=16)$ & \\
\hline Small-worldness & $1.63(0.46)$ & $1.89(0.66)$ & 0.04 & $1.55(0.34)$ & $1.84(0.51)$ & 0.02 \\
\hline Characteristic path length & $0.98(0.37)$ & $1.12(0.19)$ & 0.28 & $1.32(0.42)$ & $0.96(0.15)$ & 0.01 \\
\hline Local efficiency & $0.34(0.03)$ & $0.22(0.09)$ & 0.26 & $0.59(0.38)$ & $0.27(0.05)$ & $<0.01$ \\
\hline Global efficiency & $0.21(0.05)$ & $0.24(0.01)$ & 0.64 & $0.17(0.03)$ & $0.25(0.01)$ & 0.45 \\
\hline
\end{tabular}

cognitive dysfunction; the connectome properties of these regions may to some extent predict neurocognitive functioning [15]. Therefore, this study's findings provide new insights into the mechanism of cognitive dysfunction in cancer patients.

Evaluating the relative importance of brain neuroimaging features and their association with neurocognitive function was essential in understanding specific brain functional network patterns involved in neurocognitive dysfunction [15]. Rs-fMRI may be a particularly promising tool in identifying cancer patients at risk of long-term cancer-related brain injury $[14,15]$. In addition, connectome metrics derived from rs-fMRI show good test-retest reliability [40]. Furthermore, the rs-fMRI acquisition required approximately eight minutes, making this scan a practical possibility in busy clinical settings. Thus, utilizing rs-fMRI could be a promising tool to better understand the longitudinal changes of treatment-related neurocognitive outcomes and functional network connectome properties.
The main limitation of this study is the small sample size, which may have reduced its power to detect functional differences between patients and healthy controls. This study found limited group differences achieving statistically significant differences in neurocognitive test performance, which may partially be due to limited power. Hence, in future research, there is a need to recruit larger sample sizes and use longer-term follow-up to replicate these results, and to investigate the potential reversibility of chemotherapy-induced changes [41]. In addition, the nongeneralizable convenience sample of this study may cause potential biases that could influence the conclusions. Finally, this study only chose the AAL atlas with 90 regions (ALL-90) as a brain parcellation scheme to calculate functional connectome properties, while excluding other brain parcellation schemes, such as Harvard-Oxford Atlas, as well as randomly parceling the brain into 1024 ROIs. According to previous studies on chemotherapy-related cognitive impairment in cancer patients $[14,15,41]$, the

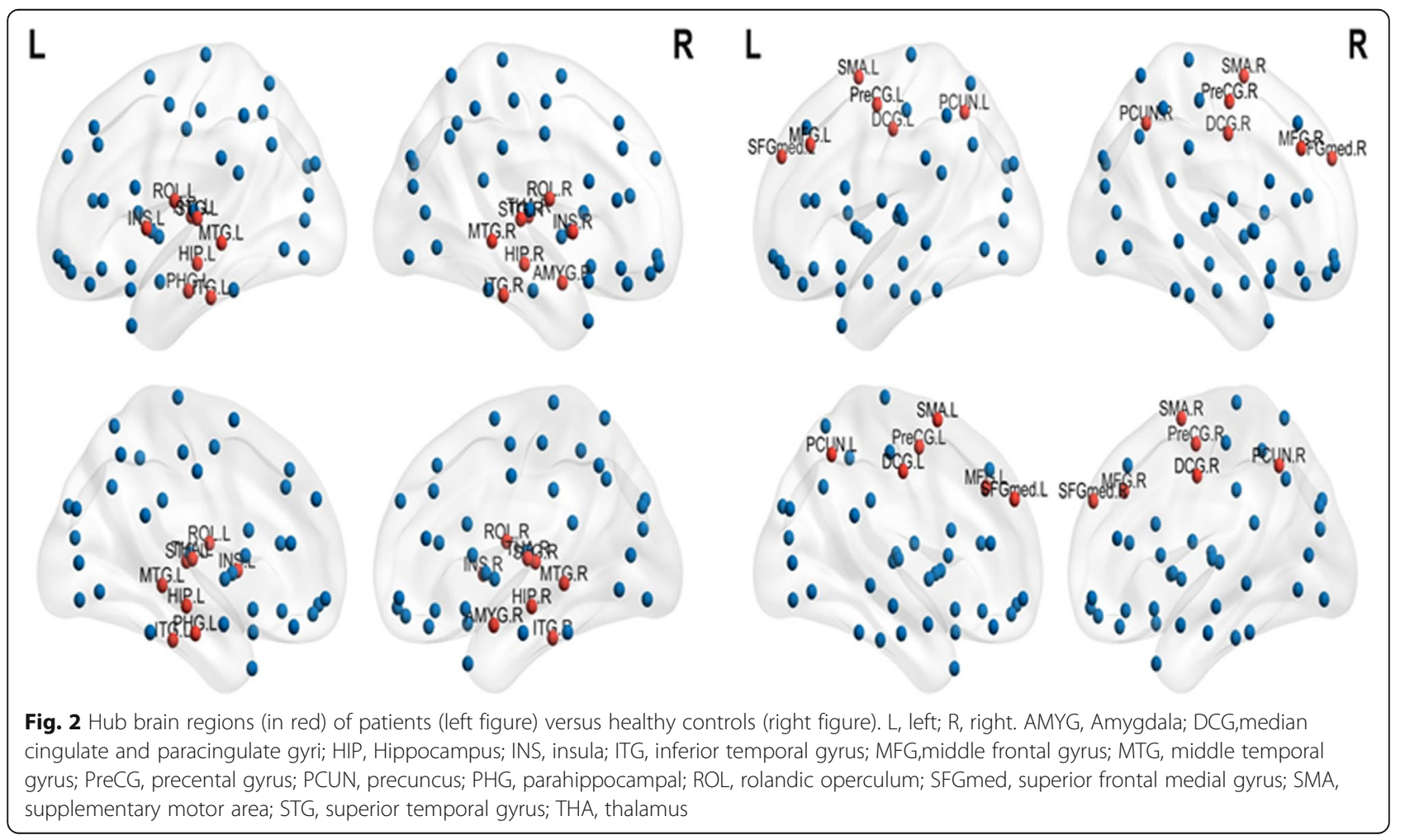




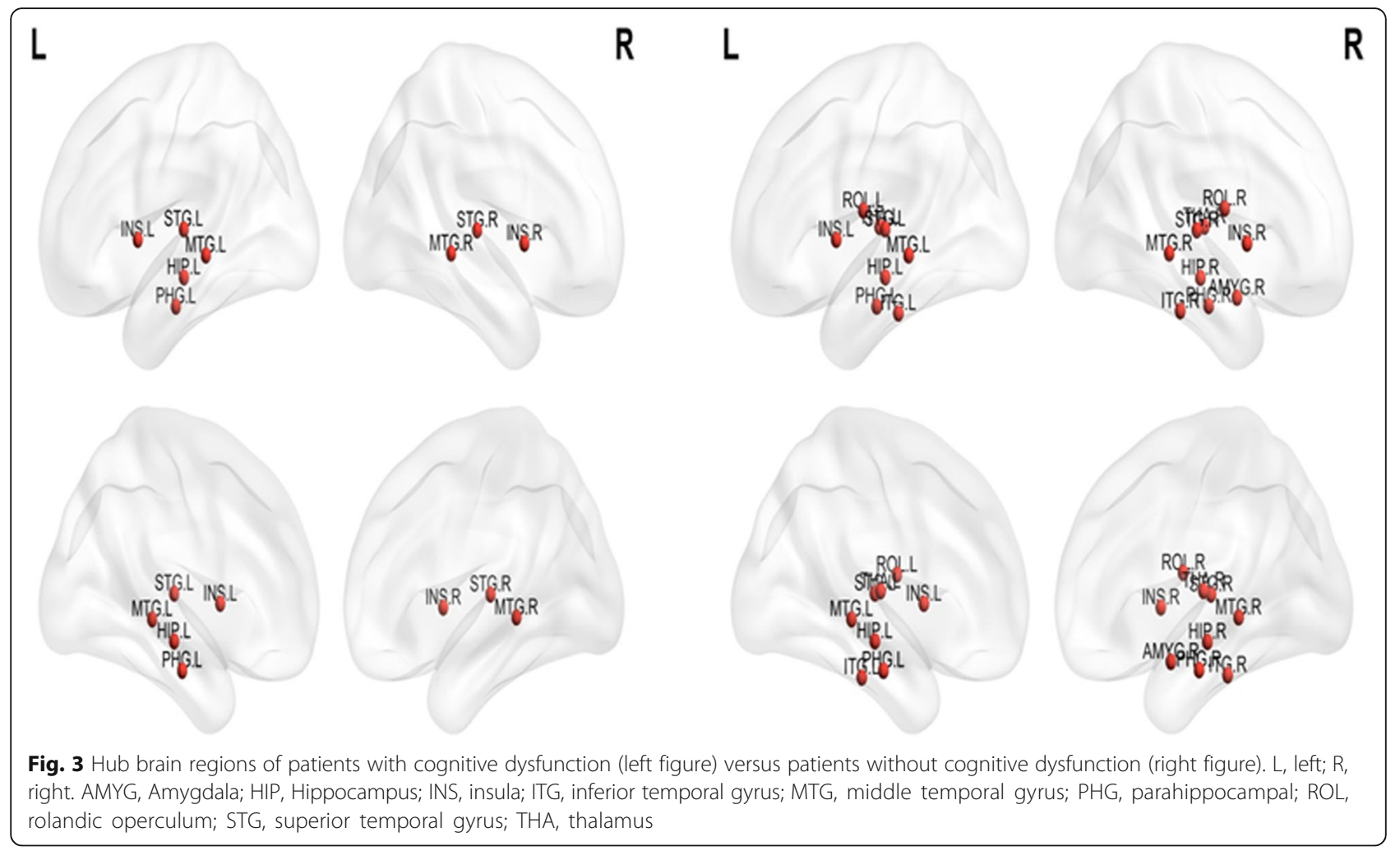

AAL-90 parcellation is one of the most common brain parcellation schemes.

\section{Conclusions}

Findings of this study have reported the first longitudinal evidence of brain functional network alteration and neurocognitive changes in Chinese gynecological cancer patients. This study found that information on the risk of brain function and neurocognitive changes following chemotherapy could potentially serve as a guide to patients in making appropriate treatment decisions, and this study may identify a cohort that could be suited for study of an intervention.

\section{Abbreviations}

AVLT-R: Auditory Verbal Learning Test - revised version; COWA: Controlled Oral Word Association Test; CRCl: Cancer-Related Cognitive Impairment; GRETNA: Graph Theoretical Network Analysis; HVLT-R: Hopkins Verbal Learning Test - revised; MRI: Magnetic Resonance Imaging; TMT: Trail Making Test

\section{Acknowledgements}

None.

\section{Funding}

This study was supported by the National Natural Science Foundation of China (No. 81872504). The funding body played the role in data collection of this study.

\section{Availability of data and materials}

Relevant data can be provided on appropriate request from the first author.

\section{Authors' contributions}

YZ, AC, and CC designed this study; YZ and AC drafted this manuscript; TS, $\mathrm{XS}, J \mathrm{X}, \mathrm{YQ}$ and $\mathrm{YZ}$ conducted this study and collected the data; $\mathrm{AC}, \mathrm{HC}$ and CC provided essential revision of this manuscript. All authors read and approved the submission of this manuscript.

\section{Ethics approval and consent to participate}

The present study was approved by the ethics committee of The Hong Kong Polytechnic University and the Third Affiliated Hospital of Guangzhou Medical University. All research participants joined this study voluntarily and provided written informed consent. All procedures performed in this study involved human subjects in accordance with the ethical standards of the ethics committee, and with the 1964 Helsinki declaration and its later amendments or comparable ethical standards.

\section{Consent for publication}

Not applicable.

\section{Competing interests}

The authors declare that they have no competing interests.

\section{Publisher's Note}

Springer Nature remains neutral with regard to jurisdictional claims in published maps and institutional affiliations.

\section{Author details}

${ }^{1}$ Research Institute of Gynecology and Obstetrics, The Third Affiliated Hospital of Guangzhou Medical University, Guangzhou, China. ${ }^{2}$ Department of Rehabilitation Sciences, The Hong Kong Polytechnic University, Hung Hom, Hong Kong, China. ${ }^{3}$ Department of Radiology, The Third Affiliated Hospital of Guangzhou Medical University, Guangzhou, China. ${ }^{4}$ Department of Oncology, The Second Affiliated Hospital of Anhui Medical University, Hefel, China. ${ }^{5}$ Department of Surgery, The Third Xiangya Hospital of Central South University, Changsha, China. 
Received: 5 August 2018 Accepted: 3 April 2019

Published online: 25 April 2019

\section{References}

1. Chen WQ, Li H, Sun KX, Zheng RS, Zhang SW, Zeng HM, Zou XN, Gu XY, He J. Report of Cancer incidence and mortality in China, 2014. Zhonghua Zhong Liu Za Zhi. 2018;40(1):5-13. https://doi.org/10.3760/cma.j.issn.0253-3766.2018.01.002.

2. Wefel JS, Kesler SR, Noll KR, Schagen SB. Clinical characteristics, pathophysiology, and management of noncentral nervous system cancer-related cognitive impairment in adults. CA Cancer J Clin. 2015;65(2):123-38. https://doi.org/10. 3322/caac.21258.

3. Joly F, Giffard B, Rigal O, De Ruiter MB, Small BJ, Dubois M, LeFel J, Schagen SB, Ahles TA, Wefel JS, Vardy JL, Pancré V, Lange M, Castel H. Impact of Cancer and its treatment on cognitive function: advances in research from the Paris international cognition and Cancer task force symposium and update since 2012. J Pain Symptom Manag. 2015;50(6):830-41. https://doi. org/10.1016/j.jpainsymman.2015.06.019.

4. Craig CD, Monk BJ, Farley JH, Chase DM. Cognitive impairment in gynecologic cancers: a systematic review of current approaches to diagnosis and treatment. Support Care Cancer. 2014;22(1):279-87. https://doi.org/10.1007/s00520-013-2029-7.

5. Ramalho M, Fontes F, Ruano L, Pereira S, Lunet N. Cognitive impairment in the first year after breast cancer diagnosis: a prospective cohort study. Breast. 2017;32:173-8. https://doi.org/10.1016/j.breast.2017.01.018.

6. Deprez S, Kesler SR, Saykin AJ, Silverman DHS, de Ruiter MB, McDonald BC. International cognition and Cancer task force recommendations for neuroimaging methods in the study of cognitive impairment in nonCNS Cancer patients. J Natl Cancer Inst. 2018;110(3):223-31. https://doi. org/10.1093/jnci/djx285.

7. Correa DD, Root JC, Kryza-Lacombe M, Mehta M, Karimi S, Hensley ML, Relkin N. Brain structure and function in patients with ovarian cancer treated with first-line chemotherapy: a pilot study. Brain Imaging Behav. 2017;11(6):1652-63. https://doi.org/10.1007/s11682-016-9608-4.

8. Zeng YC, Cheng ASK, Song T, Sheng XJ, Zhang Y, Liu XY, Chan CCH. Cognitive complaints and brain structural networks in Chinese gynaecological cancer survivors compared with age-matched controls: a cross-sectional study. BMC Cancer. 2017;17:796. https://doi.org/10.1186/s12885-017-3793-4.

9. Vannorsdall TD. Cognitive changes related to Cancer therapy. Med Clin North Am. 2017;101(6):1115-34. https://doi.org/10.1016/j.mcna.2017.06.006.

10. Cheng H, Li W, Gong L, Xuan H, Huang Z, Zhao H, Wang LS, Wang K. Altered resting-state hippocampal functional networks associated with chemotherapy-induced prospective memory impairment in breast cancer survivors. Sci Rep. 2017;7:45135. https://doi.org/10.1038/srep45135.

11. Mayeux R. Early Alzheimer's disease. N Engl J Med. 2010;362:2194-201.

12. Noonan MP, Mars RB, Sallet J, Dunbar RIM, Fellows LK. The structural and functional brain networks that support human social networks. Behav Brain Res. 2018;S0166-4328(17):31087-2. https://doi.org/10.1016/j.bbr.2018.02.019.

13. Meier J, Tewarie P, Hillebrand A, Douw L, van Dijk BW, Stufflebeam SM, Van Mieghem P. A mapping between structural and functional brain networks. Brain Connect. 2016;6(4):298-311. https://doi.org/10.1089/brain.2015.0408.

14. Kesler SR, Adams M, Packer M, Rao V, Henneghan AM, Blayney DW, Palesh $\mathrm{O}$. Disrupted brain network functional dynamics and hyper-correlation of structural and functional connectome topology in patients with breast cancer prior to treatment. Brain Behav. 2017;7(3):e00643. https://doi.org/10. 1002/brb3.643.

15. Kesler SR, Rao A, Blayney DW, Oakley-Girvan IA, Karuturi M, Palesh O. Predicting long-term cognitive outcome following breast Cancer with pre-treatment resting state fMRI and random Forest machine learning. Front Hum Neurosci. 2017;11:555. https://doi.org/10.3389/fnhum.2017.00555.

16. Bruno J, Hosseini SM, Kesler S. Altered resting state functional brain network topology in chemotherapy-treated breast cancer survivors. Neurobiol Dis. 2012;48(3):329-38

17. Fox MD, Raichle ME. Spontaneous fluctuations in brain activity observed with functional magnetic resonance imaging. Nat Rev Neurosci. 2007;8(9):700-11.

18. Kelly C, Biswal BB, Craddock RC, Castellanos FX, Milham MP. Characterizing variation in the functional connectome: promise and pitfalls. Trends Cogn Sci. 2012;16(3):181-8. https://doi.org/10.1016/j.tics.2012.02.001.

19. Shen $Y$, Yao J, Jiang $X$, Zhang L, Xu L, Feng R, Cai L, Liu J, Wang J, Chen W. Sub-hubs of baseline functional brain networks are related to early improvement following two-week pharmacological therapy for major depressive disorder. Hum Brain Mapp. 2015;36(8):2915-27. https://doi.org/10.1002/hbm.22817.
20. Tsvetanov KA, Henson RN, Tyler LK, Razi A, Geerligs L, Ham TE, Rowe JB. Cambridge Centre for Ageing and Neuroscience. Extrinsic and intrinsic brain network connectivity maintains cognition across the lifespan despite accelerated decay of regional brain activation. J Neurosci. 2016;36(11): 3115-26. https://doi.org/10.1523/JNEUROSCI.2733-15.2016.

21. Correa DD, Zhou Q, Thaler HT, Maziarz M, Hurley K, Hensley ML. Cognitive functions in long-term survivors of ovarian cancer. Gynecol Oncol. 2010; 119(2):366-9. https://doi.org/10.1016/j.ygyno.2010.06.023.

22. Hess LM, Chambers SK, Hatch K, Hallum A, Janicek MF, Buscema J, Borst M, Johnson C, Slayton L, Chongpison Y, Alberts DS. Pilot study of the prospective dentification of changes in cognitive function during chemotherapy treatment for advanced ovarian cancer. J Support Oncol. 2010;8(6):252-8.

23. Hess LM, Huang HQ, Hanlon AL, Robinson WR, Johnson R, Chambers SK, Mannel RS, Puls L, Davidson SA, Method M, Lele S, Havrilesky L, Nelson T, Alberts DS. Cognitive function during and six months following chemotherapy for front-line treatment of ovarian, primary peritoneal or fallopian tube cancer: an NRG oncology/gynecologic oncology group study. Gynecol Oncol. 2015; 139(3):541-5. https://doi.org/10.1016/j.ygyno.2015.10.003.

24. Zeng Y, Cheng ASK, Song T, Sheng X, Wang S, Xie J, Chan CCH. Effects of acupuncture on cancer-related cognitive impairment in Chinese gynecological cancer patients: a pilot cohort study. Integr Cancer Ther. 2018;17(3):737-46. https://doi.org/10.1177/1534735418777109.

25. Wefel JS, Vardy J, Ahles T, Schagen SB. International cognition and Cancer task force recommendations to harmonise studies of cognitive function in patients with cancer. Lancet Oncol. 2011;12(7):703-8. https://doi.org/10. 1016/S1470-2045(10)70294-1.

26. Guo QH. Handbook of neuropsychological assessment. 2nd ed. Shanghai: Shanghai Science and Technology Publishing Co., Ltd; 2016.

27. Strauss E, Sherman EM, Spreen OA. Compendium of Neuropsychological Tests: Administration, Norms, and Commentary. 3rd ed. NewYork: Oxford University Press; 2006.

28. Zeng YC, Cheng ASK, Liu XY, Chan CCH. Cognitive complaints and supportive care needs in Chinese cervical cancer survivors: a qualitative study. BMJ Open. 2017;7(6):e014078.

29. Wechsler D. Wechsler Intelligence Scale for Children, 4th ed. San Antonio: Psychological Corporation; 2003.

30. Wang J, Wang X, Xia M, Liao X, Evans A, He Y. GRETNA: a graph theoretical network analysis toolbox for imaging connectomics. Front Hum Neurosci. 2015;9:386. https://doi.org/10.3389/fnhum.2015.00386.

31. Cao M, He Y, Dai Z, Liao X, Jeon T, Ouyang M, Chalak L, Bi Y, Rollins N, Dong $\mathrm{Q}$, Huang $\mathrm{H}$. Early development of functional network segregation revealed by Connectomic analysis of the preterm human brain. Cereb Cortex. 2017;27(3):1949-63. https://doi.org/10.1093/cercor/bhw038.

32. Xia M, Wang J, He Y. BrainNet viewer: a network visualization tool for human brain Connectomics. PLoS One. 2013:8:e68910.

33. Sporns O, Honey C, Kötter R. Identification and classification of hubs in brain networks. PLoS One. 2017;2:e1049. https://doi.org/10.1371/journal.pone.0001049.

34. Murphy K, Birn RM, Handwerker DA, Jones TB, Bandettini PA. The impact of global signal regression on resting state correlations: are anti-correlated networks introduced? Neuroimage. 2009;44:893-905.

35. Horowitz TS, Suls J, Treviño M. A call for a neuroscience approach to Cancer-related cognitive impairment. Trends Neurosci. 2018;41(8):493-6.

36. Bullmore E, Sporns O. Complex brain networks: graph theoretical analysis of structural and functional systems. Nat Rev Neurosci. 2009;10(3):186-98. https://doi.org/10.1038/nrn2575.

37. Latora V, Marchiori M. Efficient behavior of small-world networks. Phys Rev Lett. 2001:87(19):198701.

38. Vértes PE, Bullmore ET. Annual research review: growth connectomics-the organization and reorganization of brain networks during normal and abnormal development. J Child Psychol Psychiatry. 2015;56(3):299-320. https://doi.org/10.1111/jcpp.12365.

39. van den Heuvel MP, Sporns O. Network hubs in the human brain. Trends Cogn Sci. 2013;17(12):683-96. https://doi.org/10.1016/j.tics.2013.09.012.

40. Termenon M, Jaillard A, Delon-Martin C, Achard S. Reliability of graph analysis of resting state fMRI using test-retest dataset from the human connectome project. Neuroimage. 2016;142:172-87. https://doi.org/10. 1016/j.neuroimage.2016.05.062.

41. Amidi A, Hosseini SMH, Leemans A, Kesler SR, Agerbæk M, Wu LM, Zachariae R. Changes in brain structural networks and cognitive functions in testicular cancer patients receiving cisplatin-based chemotherapy. J Natl Cancer Inst. 2017;109(12). https://doi.org/10.1093/jnci/djx085. 